\title{
Molecular impacts of rapamycin-based drug combinations: Combining rapamycin with gemcitabine or imatinib mesylate (Gleevec) in a human leiomyosarcoma model
}

\author{
OFER MERIMSKY ${ }^{1}$, YAARA GORZALCZANY ${ }^{2}$ and RONIT SAGI-EISENBERG ${ }^{2}$ \\ ${ }^{1}$ Unit of Bone and Soft Tissue Oncology, Division of Oncology, The Tel-Aviv Sourasky Medical Center, ${ }^{2}$ Department of \\ Cell and Developmental Biology, Sackler School of Medicine, Tel Aviv University, Tel Aviv 69978, Israel
}

Received February 12, 2007; Accepted April 10, 2007

\begin{abstract}
Drug combinations may provide a therapeutic benefit in treating cancer patients. However when considering a drug combination, it is important to assess how the molecular impact of the combination relates to the effects manifested by each drug alone and whether or not it varies depending on the tumor type. In this study, we have analyzed the molecular impact on a human leiomyosarcoma cell line (SK-LMS-1) of a combination consisting of the mTOR inhibitor rapamycin and either the anti-metabolite drug gemcitabine (Gemzar) or the protein tyrosine kinase inhibitor imatinib mesylate (Gleevec, STI571). We show that imatinib mesylate depolarizes the mitochondrial membrane potential $(\Delta \Phi \mathrm{m})$ and inhibits protein tyrosine phosphorylation, but displays only minor effects on cell proliferation when added alone or in combination with rapamycin. Gemcitabine or rapamycin, when added alone, inhibit protein tyrosine phosphorylation as well as phosphorylation of the MAP kinases ERK1/2. Both drugs also affect the cell cycle, arresting the cells at the S or G1 phase respectively. Rapamycin elevates significantly $\Delta \Phi \mathrm{m}$ but produces only a moderate effect on cell growth. Gemcitabine inhibits considerably cell growth but exerts no effect on $\Delta \Phi m$. Combining gemcitabine and rapamycin produces a major effect on the cell cycle, elevates the $\Delta \Phi \mathrm{m}$ even further and maintains the molecular impacts exerted by each single drug. Therefore, consistent with our clinical observation, these results suggest that combining gemcitabine and rapamycin may be beneficial in treating leiomyosarcoma patients.
\end{abstract}

Correspondence to: Dr Ronit Sagi-Eisenberg, Department of Cell and Developmental Biology, Sackler School of Medicine, Tel Aviv University, Tel Aviv 69978, Israel

E-mail: histol3@post.tau.ac.il

Abbreviations: ERK, extracellular regulated kinase; JNK, Jun kinase; MAPK, mitogen activated protein kinase; pTyr, phosphotyrosine

Key words: rapamycin, gemcitabine, imatinib mesylate (Gleevec), leiomyosarcoma, signaling

\section{Introduction}

Inherited or acquired chemoresistance are major concerns when treating cancer patients with chemotherapeutic drugs. In particular, in many occasions, the responsiveness to the chemotherapy is hindered due to the loss or interruption of apoptotic signaling pathways (1). One approach to confront this problem is to replace single drug treatments with drug combinations that include a drug directed against cellular survival-signaling networks. In this context, rapamycin has emerged as an attractive candidate. While originally used as a potent immunosuppressant (2), rapidly accumulating data implicate rapamycin as a potential anti-cancer agent $(3,4)$. mTOR, the ultimate cellular target of rapamycin belongs to the family of phosphatidylinositol-3-kinase (PI3K)-related kinases (PIKK) that control essential cell functions, including cell cycle progression, cell cycle checkpoints, DNA repair, and DNA recombination (5). Moreover, mTOR is a downstream component in the PI3K/Akt pathway, which regulates cell proliferation, cell survival and adhesion-independent survival and migration $(6,7)$. Therefore, through the inhibition of mTOR, rapamycin may display antiproliferative activity both as a single agent as well as synergistically as part of a drug combination. However when considering drug combinations, several questions need to be addressed. For example, would the molecular impact of a drug be the same irrespective of the nature of its joining drug in the combination? Does the molecular impact depend on the nature of the tumor and its genetic profile? Would the molecular impact depend on the schedule of the drug administration? Therefore, towards the broad issue of drug combinations it is important to begin addressing these questions in a systematic manner. This is of crucial importance to enable synergism rather than antagonism between the combined drugs. Towards this goal, elucidating the molecular impacts of drug combinations on human cancer cell line models should allow integration of all relevant data into a predictive model, which will serve as a guideline for future clinical regimens. To this end, in this study, we have chosen to focus on SK-LMS-1 cells as a model for leiomyosarcoma. This highly malignant neoplasm exhibits high rates of local recurrence and distant metastasis, to which the arsenal of active chemotherapy is rather limited with no standard second-line chemotherapy currently available for 
patients whose disease progressed on first-line treatment. Two rapamycin-based drug combinations were tested. The first included the anti-metabolite drug gemcitabine (Gemzar) (8), which was shown to be effective in achieving stabilization or even a small response (5.5\%) in soft tissue sarcoma (STS) patients $(9,10)$; however, it is often associated with inherited or acquired resistance (11-13). An impressive response was obtained in a leiomyosarcoma patient who was treated with such a combination (14). The second included Imatinib mesylate (Gleevec) because SK-LMS-1 cells express the receptor for PDGF (15), against which Imatinib mesylate, a protein tyrosine kinase inhibitor, displays activity $(16,17)$. However, treatment with Imatinib mesylate also involves a rapid onset of resistance resulting in a subsequent relapse. Here we describe the distinct molecular consequences that are associated with these drug combinations.

\section{Materials and methods}

Materials. Antibodies used included: polyclonal anti-phospho Akt (Ser473) and polyclonal anti-Akt (Cell signaling, Beverly, MA); monoclonal anti-phosphotyrosine 4G10 (Upstate Biotechnology, Lake Plasid, NY); monoclonal anti-diphosphorylated ERK1/2 (Sigma chemicals Co., St Louis, MO); polyclonal anti-ERK2 (SC-154, Santa Cruz Biotechnology, Inc, Santa Cruz, CA); horseradish peroxidase (HRP)-conjugated anti-mouse IgG or goat anti-rabbit IgG (Jackson Immuno Research Laboratories, West Grove, PA).

Reagents. Gemcitabine (Eli Lilly, Indianapolis, USA) was purchased from a local pharmacy. Rapamycin was purchased from LC Laboratories (USA). Capsules of imatinib mesylate (Novartis, Switzerland) were purchased from a local pharmacy. MTT (5,5',6,6'-tetrachloro-1,1',3,3-tetraethylbenzimidazolycarcocyanine iodide) was from Sigma and JC-1 was from Molecular probes (Invitrogen, Eugene, OR).

Cell culture. SK-LMS-1 cells (ATCC, Rockville, MD) were grown in DMEM supplemented with $10 \%$ FCS in a humidified atmosphere of $5 \% \mathrm{CO}_{2}$ at $37^{\circ} \mathrm{C}$.

Protein extraction and Western blotting. Samples (normalized according to protein content or number of cells) were separated on SDS-PAGE using 10\% polyacrylamide gels (BioRad) and electrophoretically (90 min at $50 \mathrm{~mA} / \mathrm{gel})$ transferred to nitrocellulose or PVDF membrane (Schleicher \& Schuell). Blots were blocked for at least $1 \mathrm{~h}$ in TBST $(10 \mathrm{mM}$ Tris- $\mathrm{HCl}$, $\mathrm{pH} 8.0,150 \mathrm{mM} \mathrm{NaCl}$, and $0.05 \%$ Tween-20) containing 5\% skim milk or $2 \%$ BSA followed by at least 1-h incubation at room temperature or overnight at $4^{\circ} \mathrm{C}$ with the desired primary antibodies. Blots were washed three times with TBST and incubated for $1 \mathrm{~h}$ at room temperature with the secondary antibodies (HRP-conjugated goat anti-rabbit or anti-mouse IgG; Jackson Research Labs). Immunoreactive bands were visualized by the enhanced chemiluminescence method.

MTT cell proliferation assay. Cells were seeded at $2 \times 10^{4} /$ well in 96-well plates in a final volume of $100 \mu 1$ and treated with the desired drug or drug combination for 2, 4, 7 and 10 days. Ten $\mu 1$ of MTT $(5 \mathrm{mg} / \mathrm{ml})$ was then added to each well

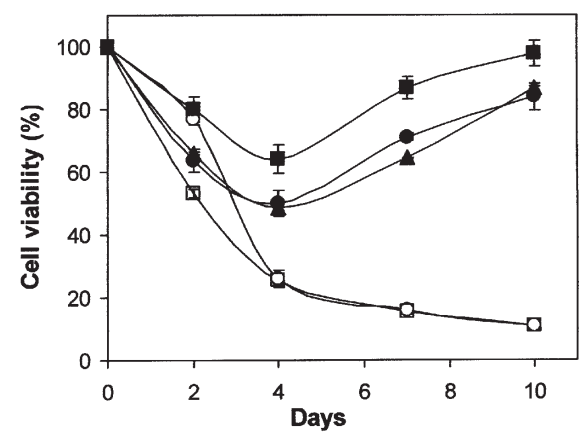

Figure 1. Modulation of cell proliferation by rapamycin, gemcitabine, imatinib mesylate and their combinations. Cells were either left untreated or treated with rapamycin $(300 \mathrm{ng} / \mathrm{ml})(\bullet)$, gemcitabine $(10 \mathrm{nM})(\circ)$, imatinib mesylate $(4 \mu \mathrm{M})(\mathbf{\square})$, gemcitabine plus rapamycin $(\square)$, or imatinib mesylate plus rapamycin $(\mathbf{\Delta})$. At the indicated time periods, cell viability was assessed by the MTT assay as described under Materials and methods. The results are presented as percentage of the O.D. of untreated cells on the same day. The data points are means \pm SEM of four determinations.

and incubated for $4 \mathrm{~h}$ at $37^{\circ} \mathrm{C}$. The reaction was blocked by the addition of $110 \mu \mathrm{l}$ of $\mathrm{HCl} 0.07 \mathrm{M}$ diluted in isopropanol and O.D. was measured at $560 \mathrm{~nm}$ using a SpectraMax 190 microplate reader (Molecular probes).

Detection of mitochondrial membrane potential ( $\Delta \Phi m)$. Mitochondrial membrane potential was analyzed essentially as described (18). Briefly, SK-LMS-1 cells were incubated with the mitochondrial membrane potential-sensitive dye JC-1 (Molecular probes) at a final concentration of $10 \mu \mathrm{M}$ in $0.8 \mathrm{ml}$ pre-warmed DMEM medium and incubated in the dark for 60 min. CCCP (Sigma) was added immediately in staining medium at a $5-\mu \mathrm{M}$ final concentration and used as a positive control for mitochondrial depolarization. The stained cells were washed, trypsinized, washed again and resuspended in $1 \mathrm{ml}$ of PBS and analyzed by FACS (FACSort; Becton Dickinson, Franklin Lakes, NJ) with an argon laser with excitation at $488 \mathrm{~nm}$ and using filters transmitting at $525 \pm 20 \mathrm{~nm}$ in the FL1 channel (green) and $590 \mathrm{~nm}$ in the FL2 channel (red). The ratio of the red (emission at $593 \mathrm{~nm}$ ) to green (emission at $532 \mathrm{~nm}$ ) fluorescence intensities was calculated for each cell.

Cell cycle. Following the desired treatments, cells were trypsinized and washed 3 times with cold PBS and resuspended in $1.0 \mathrm{ml}$ of hypotonic buffer containing $50 \mu \mathrm{g} / \mathrm{ml}$ of propidium iodide, $0.1 \%$ sodium citrate and $0.1 \%$ Triton X-100. Cells were incubated for at least $1 \mathrm{~h}$ at $4^{\circ} \mathrm{C}$ in the dark. Cells were subsequently analyzed by a fluorescence-activated sorter (FACSort). Cell cycle profiles were analyzed using WinMDI 2.8 software.

Determination of protein tyrosine phosphorylation. Cell extracts prepared by the addition of a lysis buffer containing $150 \mathrm{mM}$ sucrose, $80 \mathrm{mM}$ ß-glycerophosphate, $2 \mathrm{mM}$ EDTA, $2 \mathrm{mM}$ EGTA, $2 \mathrm{mM}$ sodium orthovanadate, $10 \mathrm{mM}$ sodium pyrophosphate, $1 \%$ Triton $\mathrm{X}-100,1 \mathrm{mM}$ phenylmethylsulfonyl fluoride and $40 \mu \mathrm{l} / \mathrm{ml}$ of the protease inhibitor cocktail, were centrifuged for $15 \mathrm{~min}$ at $12,000 \mathrm{x} \mathrm{g}$. Supernatants were mixed with $5 \mathrm{X}$ concentrated Laemmli sample buffer, boiled for $5 \mathrm{~min}$ 

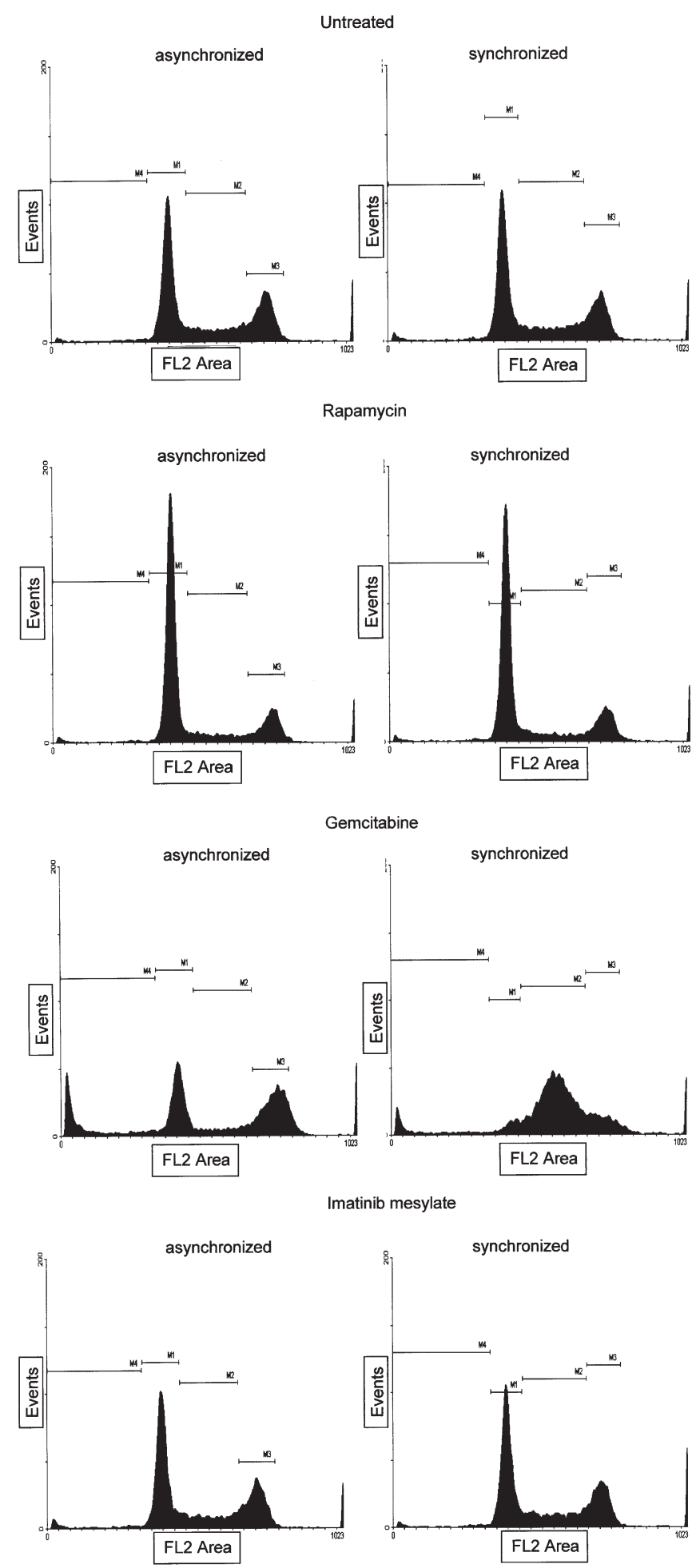

matinib mesylate

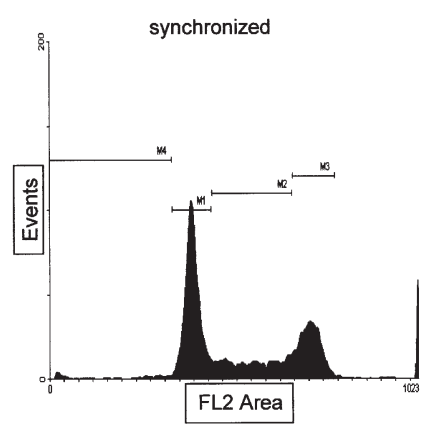

and resolved by SDS/10\% PAGE, under reducing conditions followed by transfer to PVDF membranes. The membranes were incubated overnight at $4^{\circ} \mathrm{C}$ with monoclonal antibodies, directed against P-Tyr (4G10, $0.5 \mu \mathrm{g} / \mathrm{ml})$. Bound antibodies were visualized by enhanced chemiluminescence detection with the use of goat antiserum to mouse, coupled to horseradish peroxidase (Jackson Immuno Research Laboratories).

Determination of ERK activation. Cell extracts, prepared as described above were resolved by SDS/10\% PAGE, under reducing conditions and transferred to nitrocellulose
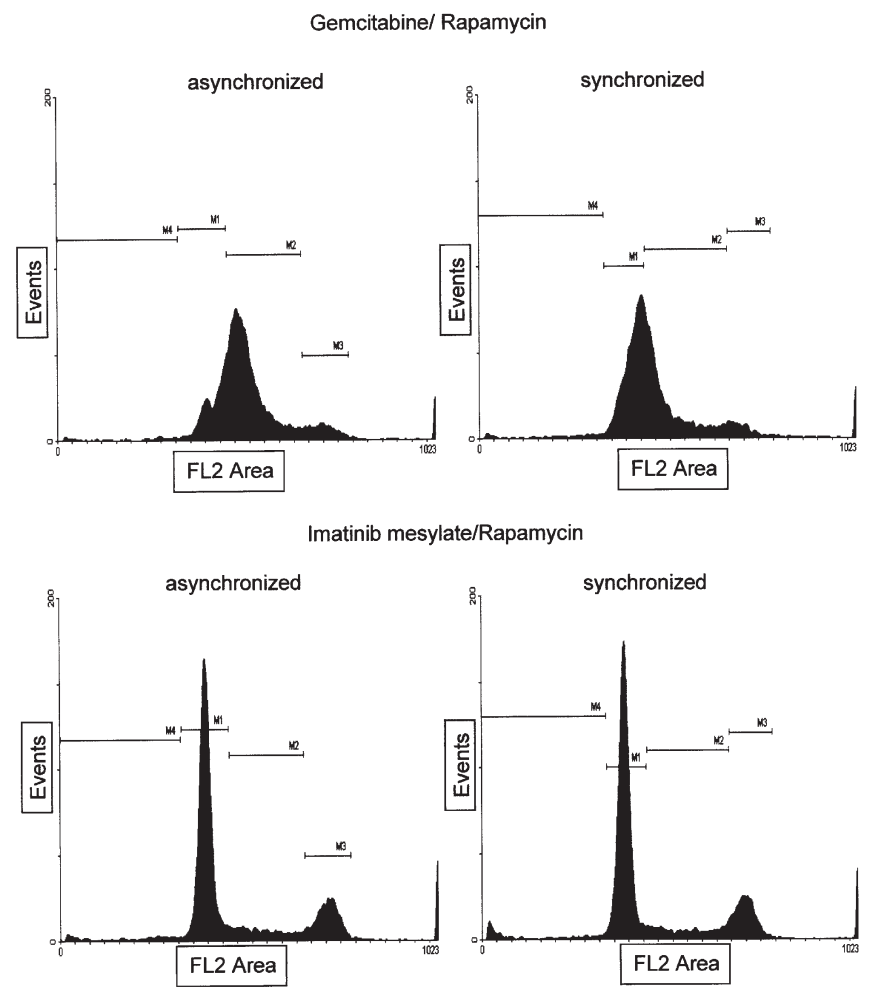

Figure 2. Modulation of the cell cycle by rapamycin, gemcitabine, imatinib mesylate and their combinations. Asynchronized cells or synchronized cells subjected to 24-h serum starvation were either left untreated or treated for $48 \mathrm{~h}$ with rapamycin $(300 \mathrm{ng} / \mathrm{ml})$, gemcitabine $(10 \mathrm{nM})$, imatinib mesylate $(4 \mu \mathrm{M})$ or combinations of imatinib mesylate and rapamycin or gemcitabine and rapamycin, as indicated. Cell cycle analyses were performed as described under Materials and methods. The numerical data are shown in Table I.

membranes. The membranes were incubated overnight at $4^{\circ} \mathrm{C}$ with polyclonal antibodies, directed against the active phosphorylated form of the p42/p44 ERK1/2 (1:20,000 dilution). Bound antibodies were visualized by enhanced chemiluminescence detection with the use of goat antiserum to rabbit, coupled to horseradish peroxidase (Jackson Immuno Research Laboratories).

Determination of Akt activation. Cell extracts, prepared as described above were resolved by SDS/10\% PAGE, under reducing conditions and transferred to nitrocellulose membranes. The membranes were incubated overnight at $4^{\circ} \mathrm{C}$ with polyclonal antibodies, directed against the active phosphorylated (S473) form of Akt. Bound antibodies were visualized by enhanced chemiluminescence detection with the use of goat antiserum to rabbit, coupled to horseradish peroxidase (Jackson Immuno Research Laboratories).

\section{Results}

Comparing the effects of rapamycin, gemcitabine, imatinib mesylate and their combinations on SK-LMS-1 cell proliferation. First we examined how rapamycin, gemcitabine and imatinib mesylate affect the proliferation of SK-LMS-1 cells, when given alone or as combinations with rapamycin. For this purpose, the number of viable cells during a ten-day treatment course was assessed by the MTT assay. As shown in Fig. 1, this assay revealed a bi-phasic effect for imatinib mesylate, 
Table I. Cell cycle analysis (\% of total).

\begin{tabular}{|c|c|c|c|c|c|c|c|c|}
\hline \multirow[b]{2}{*}{ Treatment } & \multicolumn{4}{|c|}{ Asynchronized } & \multicolumn{4}{|c|}{ Synchronized } \\
\hline & SubG0 & $\mathrm{G} 0 / \mathrm{G} 1$ & $S$ & $\mathrm{G} 2 / \mathrm{M}$ & SubG0 & G0/G1 & $\mathrm{S}$ & $\mathrm{G} 2 / \mathrm{M}$ \\
\hline None & 5.5 & 47.5 & 19.4 & 23.5 & 4.3 & 46.2 & 22.1 & 24.2 \\
\hline Rapamycin & 4.0 & 62.5 & 13.5 & 17.0 & 3.8 & 61.9 & 13.1 & 17.5 \\
\hline Gemcitabine & 19.7 & 28.9 & 11.6 & 31.9 & 9.5 & 6.9 & 62.8 & 15.3 \\
\hline Imatinib mesylate & 5.1 & 47.5 & 19.0 & 24.5 & 3.8 & 44.6 & 22.8 & 24.9 \\
\hline Rapamycin/gemcitabine & 4.0 & 23.0 & 57.4 & 12.9 & 4.5 & 43.4 & 42.2 & 6.8 \\
\hline Rapamycin/imatinib mesylate & 8.1 & 57.5 & 14.4 & 16.8 & 6.7 & 60.0 & 13.2 & 17.1 \\
\hline
\end{tabular}

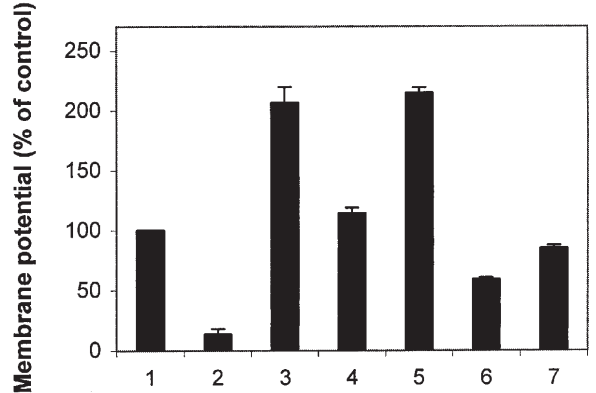

Figure 3. Effects of rapamycin, gemcitabine, imatinib mesylate and their combinations on the mitochondrial membrane potential. Cells were left untreated (UT) or treated for $48 \mathrm{~h}$ with rapamycin $(300 \mathrm{ng} / \mathrm{ml})$, gemcitabine $(10 \mathrm{nM})$, imatinib mesylate $(4 \mu \mathrm{M})$ or combinations of imatinib mesylate and rapamycin or gemcitabine and rapamycin as indicated. Cells were subsequently stained with JC-1 and analyzed for mitochondrial membrane potential by FACS as described under Materials and methods. The mitochondrial uncoupler CCCP $(5 \mu \mathrm{M})$ was added as a control to depolarize the mitochondria. A histogram of the average percentage \pm SEM of the depolarized or hyperpolarized cell populations derived from three separate experiments is presented, where $100 \%$ was designated as the ratio between red to green fluorescence intensity of untreated cells. (1) Untreated cells, (2) cells treated with CCCP $(5 \mu \mathrm{M})$, (3) cells treated with rapamycin $(300 \mathrm{ng} / \mathrm{ml})$, (4) cells treated with gemcitabine $(10 \mathrm{nM}),(5)$ cells treated with rapamycin $(300 \mathrm{ng} / \mathrm{ml})$ and gemcitabine $(10 \mathrm{nM}),(6)$ cells treated with imatinib mesylate $(4 \mu \mathrm{M})$, (7) cells treated with imatinib mesylate $(4 \mu \mathrm{M})$ and rapamycin $(300 \mathrm{ng} / \mathrm{ml})$.

whereby cell viability decreased by approximately $40 \%$ during the first 4 days of treatment, but this effect was transient and the cells regained their full viability at day 10, possibly because of degradation of the drug. Rapamycin behaved somewhat similarly, except that it reduced cell viability by $50 \%$ and only $80 \%$ viability was recorded at day 10 (Fig. 1). Gemcitabine was by far more effective leaving only $10 \%$ viability at day 10 (Fig. 1). Inspecting the effects of the drug combinations has demonstrated that while rapamycin/imatinib mesylate was equipotent to rapamycin alone, rapamycin/gemcitabine was basically similar in its effect to gemcitabine alone, except for at short periods ( $48 \mathrm{~h}$ ) when the combination of rapamycin plus gemcitabine was clearly more efficient than each drug alone (Fig. 1). Taken together, the results of this set of experiments have indicated that gemcitabine is most potent in inducing cell death of this particular cell line. In contrast, rapamycin had a moderate effect while imatinib mesylate was ineffectual. Finally, rapamycin neither synergized with nor antagonized the effects of gemcitabine or imatinib mesylate on proliferation of leiomyosarcoma cells in vitro.
Comparing the effects of rapamycin, gemcitabine, imatinib mesylate and their combinations on SK-LMS-1 cell cycle. Both gemcitabine and rapamycin are known to mediate cell cycle arrest. Specifically, rapamycin prevents G1 to S progression (19), while gemcitabine arrests cells at the $S$ phase. Imatinib mesylate is a tyrosine kinase inhibitor, which may or may not affect the cell cycle depending on its intracellular targets. Therefore, we performed cell cycle analyses of cells treated with each of these drugs alone or in combination to assess their effects on cell cycle progression of SK-LMS-1 cells. Moreover, cell cycle profiles were analyzed both in asynchronized cells, which were grown in the presence of serum, or in cells which were subjected to a 24-h serum starvation period, prior to their desired treatments. As shown in Fig. 2 and Table I, serum starvation had no effect on the cell cycle distribution. Approximately $46 \%$ of the cells were found at the G0/G1 phase, $20 \%$ in S phase and $25 \%$ in G2/M. Consistent with its reported effects on the cell cycle in other cell types, and irrespective of whether or not serum was deprived, rapamycin induced a significant (from 46 to $63 \%$ ) increase in the number of cells arrested at the G0/G1 phase (Fig. 2). In sharp contrast, the effect of gemcitabine was clearly serum-dependent. In synchronized cells, gemcitabine induced a remarkable increase (from 20 to 62\%) in S phase arrested cells (Fig. 2). However, in asynchronized cells gemcitabine produced no significant impact (Fig. 2). Imatinib mesylate displayed no effect on the cell cycle distribution (Fig. 2). Combining imatinib mesylate and rapamycin had the same impact as treating the cells with rapamycin alone, namely the fraction of cells at G1 phase of the cell cycle increased to $\sim 60 \%$ in both synchronized and asynchronized cells (Fig. 2). Strikingly, combining gemcitabine with rapamycin produced a dramatic effect on the cell cycle distribution (Fig. 2 and Table I). In asynchronized cells, the combination resulted in cell arrest at the $\mathrm{S}$ phase of the cell cycle, thus giving rise to a phenotype similar to that displayed by gemcitabine alone in synchronized cells. In contrast in synchronized cells, an equal distribution between the G0/G1 and $\mathrm{S}$ phase was recorded (Fig. 2). These analyses have therefore strongly suggested that in asynchronized SK-LMS-1 cells, the ability of gemcitabine to impose cell cycle arrest depended on the combined presence of rapamycin.

Comparing the effects of rapamycin, gemcitabine, imatinib mesylate and their combinations on the mitochondrial membrane potential. Changes in the mitochondrial membrane potential $(\Delta \Phi \mathrm{m})$ are tightly linked with cell death by either 
A

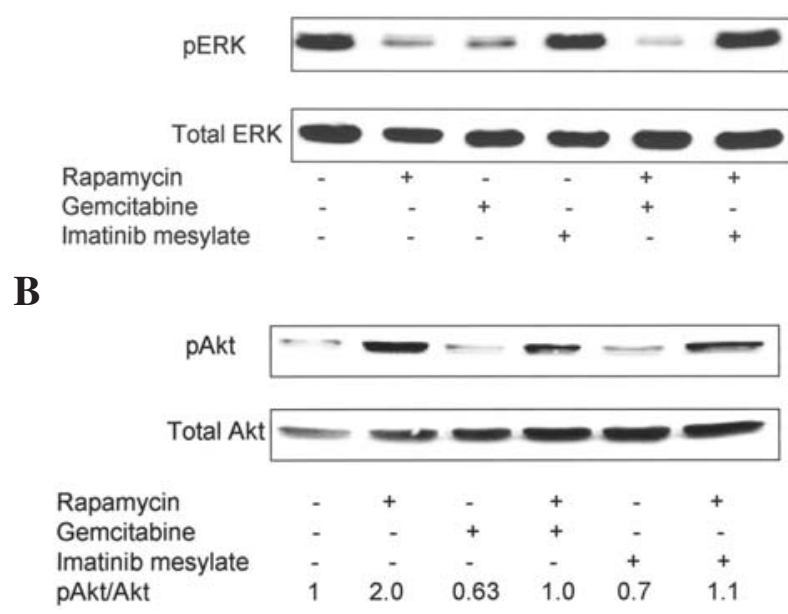

Figure 4. Inhibition of signaling kinases by rapamycin, gemcitabine, imatinib mesylate and their combinations. Cells were either left untreated or treated with the indicated drugs for 2 days. Cell lysates $(50 \mu \mathrm{g} / \mathrm{lane})$ were resolved by SDS-PAGE, subjected to Western blot analysis and probed with (A) anti-active ERK1/2 antibodies and reprobed with anti-total ERK or (B) with anti-pAkt (Ser473) and reprobed with anti-total Akt.

apoptosis $(20,21)$ or necrosis $(22)$. Therefore, we analyzed whether and how these drug combinations would affect $\Delta \Phi \mathrm{m}$. Comparing the red to green fluorescence ratio of JC-1 stained control cells and cells which were treated with gemcitabine for $48 \mathrm{~h}$ revealed no significant differences (Fig. 3), therefore indicating that this drug does not affect significantly the $\Delta \Phi \mathrm{m}$ of SK-LMS-1 cells. In contrast, both rapamycin and imatinib mesylate produced profound, yet distinct effects. Rapamycin alone, or when combined with gemcitabine elevated $\Delta \Phi \mathrm{m}$ by 2-fold (Fig. 3). Imatinib mesylate alone or with rapamycin depolarized the $\Delta \Phi m$ (Fig. 3). Notably, addition of the protonophore CCCP depolarized completely the mitochondria yielding a similar red-to-green ratio in both control and treated cells (Fig. 3).

Comparing the effects of rapamycin, gemcitabine, imatinib mesylate and their combinations on the activation state of signaling kinases. To begin dissecting and identifying the signaling networks that are affected by these drug combinations, we next explored their effects on signaling kinases known to regulate cell proliferation or survival. Specifically, we examined the activation state in control or treated cells of the MAP kinases ERK1/2, whose deregulation is often associated with cancer; JNK, whose activation is linked

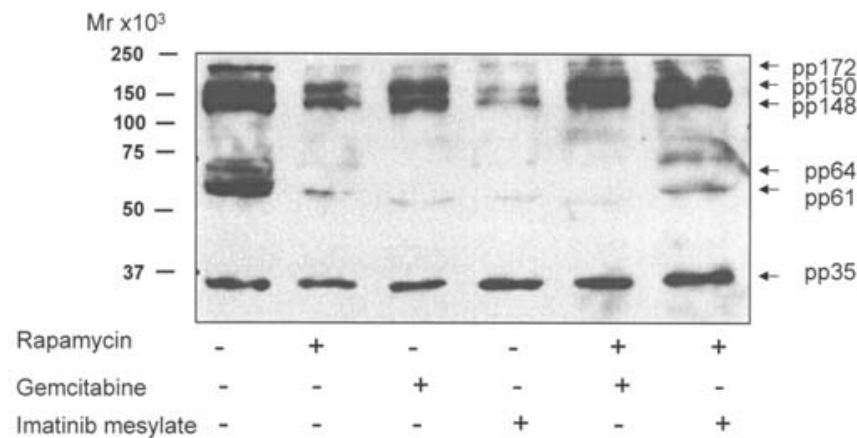

Figure 5. Inhibition of protein tyrosine phosphorylation by rapamycin, gemcitabine, imatinib mesylate and their combinations. Cells were either left untreated or treated with the indicated drugs for 2 days. Cell lysates ( $80 \mu \mathrm{g} /$ lane) were resolved by SDS-PAGE, subjected to Western blot analysis and probed with anti-phosphotyrosine antibodies. The bands corresponding to pp172, pp150/148, pp64, pp61 and pp35 were quantified by densitometry and are presented as percentage of their intensities in untreated cells.

with apoptosis; and finally, Akt, whose activation is associated with cell survival. Untreated SK-LMS-1 cells display phospho-ERK1/2, the level of which is neither affected by imatinib mesylate alone nor by its combination with rapamycin (Fig. 4A). In contrast, rapamycin or gemcitabine, when administered alone reduced the level of phospho-ERK by $50 \%$ (Fig. 4A). Their combination was even more effective, reducing significantly (by $75 \%$ ) the level of active ERK $1 / 2$ (Fig. 4A).

Next, we attempted to assess the effect of these drugs and their combinations on the state of activation of the JNKs; however, we were unable to detect any P-JNK in untreated or any of the drug-treated cells (data not shown).

Finally, Western blot analysis using antibodies directed against S473-p-Akt revealed that untreated SK-LMS-1 cells contain phosphorylated Akt, which was reduced by either gemcitabine or by imatinib mesylate (Fig. 4B). In contrast, treatment with rapamycin increased by 2 -fold the content of pAkt (Fig. 4B). Interestingly, this effect was abolished when rapamycin was combined with either gemcitabine or with imatinib mesylate (Fig. 4B).

Since imatinib mesylate is a protein tyrosine kinase inhibitor, we also compared the general profile of tyrosine phosphorylated proteins in control and treated cells. Indeed, several phosphotyrosine containing proteins could be clearly detected in untreated SK-LMS-1 cells (Fig. 5). These included proteins of Mr of 172, 148/150, 64, 61 and $35 \mathrm{kDa}$ (Fig. 5). Interestingly, with the exception of pp35, which was resistant

Table II. Protein tyrosine phosphorylation analysis (\% of control).

\begin{tabular}{lccccc}
\hline & Rapamycin & Gemcitabine & Imatinib mesylate & Rapamycin/gemcitabine & Rapamycin/imatinib mesylate \\
\hline pp172 & 20 & 40 & 40 & 85 & 73 \\
pp148/150 & 40 & 65 & 30 & 80 & 90 \\
pp64 & 25 & 10 & 20 & 25 & 93 \\
pp61 & 20 & 10 & 10 & 15 & 44 \\
pp35 & 70 & 80 & 110 & 120 & 160 \\
\hline
\end{tabular}


to all treatments, all three drugs, rapamycin, imatinib mesylate and the non-signaling, anti-metabolite drug, gemcitabine, inhibited tyrosine phosphorylation of all the remaining proteins (Fig. 5, Table II). Specifically, pp172 and pp64 were highly and similarly sensitive to all three drugs, and pp148/150 was almost entirely inhibited by imatinib mesylate and partially inhibited by rapamycin or gemcitabine (Fig. 5). In contrast, pp61 was more sensitive to imatinib mesylate or gemcitabine and less to rapamycin (Fig. 5, Table II). Strikingly, distinct phosphorylation profiles were recorded in cells subjected to the drug combinations. pp172 was inhibited to a lesser extent by either of the rapamycin-based combinations, whereas pp148/150 remained phosphorylated in the presence of rapamycin/gemcitabine or rapamycin/imatinib mesylate (Fig. 5, Table II). The combination of rapamycin/gemcitabine was as potent as each drug separately in inhibiting tyrosine phosphorylation of pp64 and pp61. However, in marked contrast, the combination of rapamycin/imatinib mesylate was much less effective in inhibiting tyrosine phosphorylation of these proteins (Fig. 5, Table II). These results have therefore indicated that the combination of rapamycin and imatinib mesylate displays antagonistic features at least with regard to the tyrosine phosphorylation of certain, yet unidentified, cellular proteins.

\section{Discussion}

Chemotherapeutic drug combinations, which include a signaling drug aimed at inhibiting survival or proliferation signaling networks, may provide a therapeutic benefit in particular when chemoresistance is encountered or induced. However, the molecular impact of each drug in combination might be influenced by its companion drug, giving rise to a complex cross-talk. Moreover, the latter may not only depend on the combination but also on the type of tumor it is to be applied to. Therefore, in vitro analyses aimed at elucidating the molecular mechanisms and resultant impact of chemotherapeutic drug combinations on model human cancer cell lines are useful tools, which should provide important information for the proper design of clinical regimens. For example, gemcitabine is currently used for treating pancreatic carcinoma (23), non-small cell lung carcinoma (NSCLC) (24), soft tissue sarcoma (25) and others. It is well established that gemcitabine is activated intracellularly by nucleoside kinases to generate active di and triphosphate nucleosides, which compete with $\mathrm{dCTP}$, therefore resulting in chain termination (8). However, the molecular impact of this drug is only partially resolved and, based on the available data, it seems to depend on the tumor type. Thus, in NSCLC, activation of the MAP kinases ERK1/2 was reported to mediate gemcitabineinduced cell death (26). In contrast, activation of ERK1/2 is linked with gemcitabine resistance in pancreatic cancer (27). Rapamycin inhibits mTOR complex 1 (mTORC1), which stimulates cell growth by modulating the activity of S6K1 and 4E-BP1 (28). Rapamycin also blocks Akt-mediated survival by inhibiting mTOR complex 2 (mTORC2) (29). However, unlike the universal inhibition of mTORC 1 by rapamycin, inhibition of mTORC2 is cell type-dependent (29). Thus, because S6K1 suppresses signaling through the PI3K/ Akt pathway, selective inhibition of mTORC1 by rapamycin may remove the inhibitory constraint exerted by S6K1 and thereby enhance Akt phosphorylation and activation, rather than blocking it (29). To add even further complexity to the system, recent studies have demonstrated that, while Akt promotes tumor progression in numerous cancer types, it blocks breast cancer cell motility and invasion (30) and its biological functions are isotype-specific (31). These findings thus signify the importance of evaluating the molecular impact of chemotherapeutic drugs on human models for distinct types of cancer prior to and towards their introduction into the clinic. In the present study, we began characterizing the molecular consequences of subjecting SK-LMS-1 human leiomyosarcoma cells to gemcitabine, rapamycin and imatinib mesylate, all of which are currently used in the clinic to treat patients with certain types of sarcoma. In particular the combination with rapamycin was chosen because, based upon our own clinical experience (14) and the reports of others (32), rapamycinbased combinations have proven beneficial in treating sarcoma patients. Indeed, rapamycin was shown to synergize with gemcitabine in a pancreatic carcinoma model (33) and with imatinib mesylate in leukemia (34), and to enhance the cytotoxic effects of radiation in breast cancer cell models (3). Herein, we show that gemcitabine and imatinib mesylate produce distinct molecular effects and incorporate differently their combination with rapamycin. Gemcitabine inhibits SKLMS-1 cell proliferation. Importantly, this effect does not involve any changes in the cell cycle progression, but is associated with molecular effects on cell signaling. Specifically, in contrast to its effect in NSCLC (26), the cytotoxic effect of gemcitabine on SK-LMS-1 cells is associated with inhibition of phosphorylation of ERK1/2, Akt and several yet unidentified tyrosine phosphorylated proteins. These results are consistent with recent reports, which demonstrated inhibition of signaling pathways by gemcitabine (35). Notably, consistent with its well-established effect on the cell cycle, gemcitabine could arrest the SK-LMS-1 cells in their S phase of the cell cycle. However, this effect was only observed in synchronized cells, which were first subjected to serum deprivation. Rapamycin is by far less effective in inhibiting proliferation of SK-LMS-1 cells as compared to gemcitabine. However, like gemcitabine, rapamycin also reduces significantly the amount of active pERK1/2 and the amount of tyrosine phosphorylated proteins. Unlike, gemcitabine, rapamycin increases the level of pAkt, therefore indicating that it fails to inhibit mTORC2. Notably, similar results were obtained in U2OS cells, a model for osteosarcoma and rhabdomyosarcoma Kym-1 cells (29), where rapamycin failed to inhibit Akt. Rapamycin also displays two additional molecular effects. First, rapamycin affects the cell cycle leading to an increased fraction of cells arrested at the G0G1 phase. Second, rapamycin elevates the mitochondrial membrane potential. The latter is presumably linked with reversed activity of the mitochondrial F0F1 ATPase caused by reduced ADP transport into the mitochondria (36). Such activity may in turn lead to ATP depletion and thereby facilitate cell death (37). Indeed, the ability of rapamycin to augment the response to radiation of the human breast cancer cell line MCF-7 is mediated by the elevation of the mitochondrial membrane potential (36). By this mechanism rapamycin may also expand the activity of gemcitabine. In agreement with this notion, in the presence of rapamycin, gemcitabine 
regains its ability to affect the cell cycle leading to remarkable $\mathrm{S}$ phase arrest. Moreover, these drugs synergize in reducing ERK1/2 activation as well as in inhibiting cell growth under conditions at which the effect of gemcitabine alone is submaximal (i.e. following $48 \mathrm{~h}$ of treatment). In addition, the stimulatory effect of rapamycin on the phosphorylation of Akt is eliminated.

Imatinib mesylate produced only a moderate effect on SK-LMS-1 cell proliferation. Although these cells express the PDGF receptor (15), we were unable to detect the receptor by immunohistochemistry (not shown), which may indicate a low expression level of this receptor in these cells. Interestingly, imatinib mesylate affected the cells as indicated both by depolarization of the mitochondrial membrane potential and inhibition of protein tyrosine as well as Akt phosphorylation. However, no indication of apoptosis was obtained by nuclei stain or caspase activation (not shown). In addition, no indication of any synergism between imatinib mesylate and rapamycin were recorded in terms of cell proliferation or cell cycle. In fact, when combined, imatinib mesylate antagonized the inhibitory effect exerted by rapamycin on ERK1/2 activation and the tyrosine phosphorylation of pp64 and pp61, two yet unidentified tyrosine phosphorylated proteins.

In summary, our results indicate the importance of the specific evaluation of the molecular impact of drug combinations for each type of cancer. The present study employing SK-LMS-1 cells as a model for human leiomyosarcoma, reveals no advantage in combining imatinib mesylate with rapamycin, although the cells do express the PDGF receptor. In contrast combining rapamycin with gemcitabine may yield a therapeutic advantage. Although gemcitabine displayed remarkable activity in this cellular model, its efficacy might be limited when treating cancer patients. Firstly, the bioavailability of the drug is considerably lower as compared with the in vitro experimental conditions where the cell line is continuously exposed to the drug and, secondly, drug resistance is not acquired under the short-term conditions of the cells in culture. It is therefore envisioned that, in vivo, the facts that rapamycin hyperpolarizes the mitochondria, synergizes with gemcitabine in inhibiting ERK1/2 activation and protein tyrosine phosphorylation and produces a major effect on the cell cycle strongly suggest that the combination of gemcitabine with rapamycin may provide a therapeutic benefit in treating leiomyosarcoma patients.

\section{Acknowledgements}

We thank Dr D. Neumann for helpful discussions and a critical reading of this manuscript. This study is dedicated to the memory of Dr Avner Eisenberg.

\section{References}

1. Pommier Y, Sordet O, Antony S, Hayward RL and Kohn KW: Apoptosis defects and chemotherapy resistance: molecular interaction maps and networks. Oncogene 23: 2934-2949, 2004.

2. Dupont $\mathrm{P}$ and Warrens $\mathrm{AN}$ : The evolving role of sirolimus in renal transplantation. QJM 96: 401-409, 2003.

3. Albert JM, Kim KW, Cao C and Lu B: Targeting the Akt/ mammalian target of rapamycin pathway for radiosensitization of breast cancer. Mol Cancer Ther 5: 1183-1189, 2006.
4. Dancey JE: Inhibitors of the mammalian target of rapamycin. Expert Opin Investig Drugs 14: 313-328, 2005.

5. Keith CT and Schreiber SL: PIK-related kinases: DNA repair, recombination, and cell cycle checkpoints. Science 270: 50-51, 1995.

6. Dancey JE: Molecular targeting: PI3 kinase pathway. Ann Oncol 15: 233-239, 2004.

7. Kim D, Cheng G, Lindsley C, Yang H and Cheng J: Targeting the phosphatidylinositol-3 kinase/Akt pathway for the treatment of cancer. Curr Opin Investig Drugs 6: 1250-1258, 2005.

8. Hui YF and Reitz J: Gemcitabine: a cytidine analogue active against solid tumors. Am J Health Syst Pharm 54: 162-170, 1997.

9. Merimsky O, Meller I, Flusser G, et al: Gemcitabine in soft tissue or bone sarcoma resistant to standard chemotherapy: a phase II study. Cancer Chemother Pharmacol 45: 177-181, 2000.

10. Patel SR, Gandhi V, Jenkins J, et al: Phase II clinical investigation of gemcitabine in advanced soft tissue sarcomas and window evaluation of dose rate on gemcitabine triphosphate accumulation. J Clin Oncol 19: 3483-3489, 2001.

11. Arlt A, Gehrz A, Müerköster S, et al: Role of NF-kappaB and Akt/PI3K in the resistance of pancreatic carcinoma cell lines against gemcitabine-induced cell death. Oncogene 22: 3243-3251, 2003.

12. Oguri T, Achiwa H, Sato S, et al: The determinants of sensitivity and acquired resistance to gemcitabine differ in non-small cell lung cancer: a role of ABCC5 in gemcitabine sensitivity. Mol Cancer Ther 5: 1800-1806, 2006.

13. Williams J, Lucas PC, Griffith KA, et al: Expression of Bcl-xL in ovarian carcinoma is associated with chemoresistance and recurrent disease. Gynecol Oncol 96: 287-295, 2005.

14. Merimsky O: Targeting metastatic leiomyosarcoma by rapamycin plus gemcitabine: an intriguing clinical observation. Int $\mathrm{J}$ Mol Med 14: 931-935, 2004

15. Loizos N, Xu Y, Huber J, et al: Targeting the platelet-derived growth factor receptor $\{$ alpha\} with a neutralizing human monoclonal antibody inhibits the growth of tumor xenografts: Implications as a potential therapeutic target. Mol Cancer Ther 4: 369-379, 2005

16. Heinrich MC, Griffith DJ, Druker BJ, Wait CL, Ott KA and Zigler AJ: Inhibition of c-kit receptor tyrosine kinase activity by STI 571, a selective tyrosine kinase inhibitor. Blood 96: 925-932, 2000.

17. Buchdunger E, Cioffi CL, Law N, et al: Abl protein-tyrosine kinase inhibitor STI571 inhibits in vitro signal transduction mediated by c-Kit and platelet-derived growth factor receptors. J Pharmacol Exp Ther 295: 139-145, 2000.

18. Salvioli S, Ardizzoni A, Franceschi C and Cossarizza A: JC-1, but not DiOC6(3) or rhodamine 123, is a reliable fluorescent probe to assess [Delta][Psi] changes in intact cells: implications for studies on mitochondrial functionality during apoptosis. FEBS Letters 411: 77-82, 1997.

19. Brown EJ, Albers MW, Shin TB, et al: A mammalian protein targeted by G1-arresting rapamycin-receptor complex. Nature 369: 756-758, 1994.

20. Green DR and Reed JC: Mitochondria and apoptosis. Science 281: 1309-1312, 1998.

21. Ly JD, Grubb DR and Lawen A: The mitochondrial membrane potential in apoptosis; an update. Apoptosis 8: 115-128, 2003.

22. Kroemer G, Dallaporta B and Resche-Rigon M: The mitochondrial death/life regulator in apoptosis and necrosis. Ann Rev Physiol 60: 619-642, 1998.

23. Cappuzzo F, Toschi L, Finocchiaro G, Bartolini S and Gioia V: Role of gemcitabine in cancer therapy. Future Oncol 1: 7-17, 2005.

24. Cappuzzo F and Crino L: Gemcitabine in non-small cell lung cancer. Expert Opin Pharmacother 3: 745-753, 2002.

25. Bauer S, Seeber S and Schutte J: Gemcitabine in the treatment of Soft Tissue Sarcomas. Onkologie 27: 180-186, 2004.

26. Chang G-C, Hsu S-L, Tsai J-R, Wu W-J, Chen C-Y and Sheu G-T: Extracellular signal-regulated kinase activation and $\mathrm{Bcl}-2$ downregulation mediate apoptosis after gemcitabine treatment partly via a p53-independent pathway. Eur J Pharmacol 502: 169-183,2004.

27. Yokoi K and Fidler IJ: Hypoxia increases resistance of human pancreatic cancer cells to apoptosis induced by gemcitabine. Clin Cancer Res 10: 2299-2306, 2004.

28. Kim D-H, Sarbassov DD, Ali SM, et al: mTOR interacts with raptor to form a nutrient-sensitive complex that signals to the cell growth machinery. Cell 110: 163-175, 2002. 
29. Sarbassov DD, Ali SM, Sengupta S, et al: Prolonged rapamycin treatment inhibits mTORC2 assembly and Akt/PKB. Mol Cell 22: 159-168, 2006.

30. Yoeli-Lerner M, Yiu GK, Rabinovitz I, Erhardt P, Jauliac S and Toker A: Akt blocks breast cancer cell motility and invasion through the transcription factor NFAT. Mol Cell 20: 539-550, 2005.

31. Stambolic V and Woodgett JR: Functional distinctions of protein kinase $\mathrm{B} / \mathrm{Akt}$ isoforms defined by their influence on cell migration. Trends Cell Biol 16: 461-466, 2006.

32. Okuno S: Mammalian target of rapamycin inhibitors in sarcomas. Sarcomas. Curr Opin Oncol 18: 360-362, 2006.

33. Bruns CJ, Koehl GE, Guba M, et al: Rapamycin-induced endothelial cell death and tumor vessel thrombosis potentiate cytotoxic therapy against pancreatic cancer. Clin Cancer Res 10: 2109-2119, 2004.
34. Mohi MG, Boulton C, Gu T-L, et al: Combination of rapamycin and protein tyrosine kinase (PTK) inhibitors for the treatment of leukemias caused by oncogenic PTKs. PNAS 101: 3130-3135, 2004.

35. Feng FY, Varambally S, Tomlins SA, et al: Role of epidermal growth factor receptor degradation in gemcitabine-mediated cytotoxicity. Oncogene 2006 (Epub ahead of print).

36. Paglin S, Lee N-Y, Nakar C, et al: Rapamycin-sensitive pathway regulates mitochondrial membrane potential, autophagy, and survival in irradiated MCF-7 cells. Cancer Res 65: 11061-11070, 2005.

37. Khaled AR, Reynolds DA, Young HA, Thompson CB, Muegge K and Durum SK: Interleukin-3 withdrawal induces an early increase in mitochondrial membrane potential unrelated to the Bcl-2 family. Roles of intracellular $\mathrm{pH}$, ADP transport, and F0F1-ATPase. J Biol Chem 276: 6453-6462, 2001. 